\title{
Diastolic dysfunction in intensive care unit: a pilot study in the general intensive care unit of L'Aquila - Italy
}

Biancofiore V. ${ }^{1}$, Salve G. ${ }^{2}$, Ciccozzi A. ${ }^{3}$, Angeletti P.M. ${ }^{4}$, Prof. Marinangeli F. ${ }^{3}$, Zugaro A. ${ }^{5}$

Institutes:

1 Università degli studi dell'Aquila sede aggregata Chieti, Dept of Anaesthesiology \& Intensive Care, L'Aquila, Italy. 2 Università degli studi dell'Aquila, L'Aquila, Italy. 3 Università degli studi dell'Aquila, Dept of Anaesthesiology \& Intensive Care, L'Aquila, Italy. 4

Ospedale Mazzini di Teramo, Dept of Anaesthesiology \& Intensive Care, Teramo, Italy. $\mathbf{5}$ Ospedale S. Salvatore di L'Aquila Dept of Anaesthesiology \& Intensive Care L'Aquila, Italy

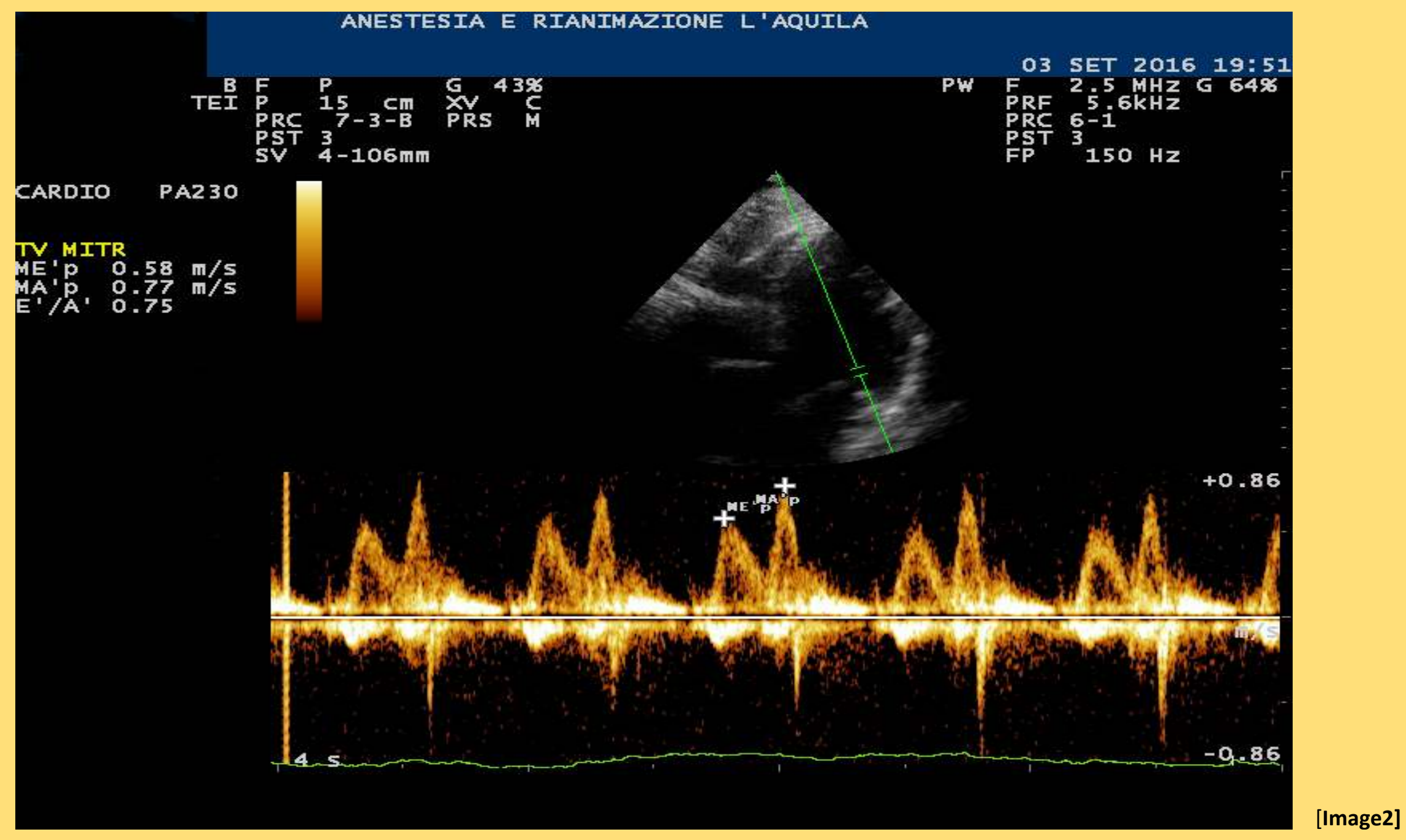

Diastolic Dysfunction (DD) is a known cause of heart failure, related to frequent systemic diseases in ICU patients; but the actual prevalence of DD in ICU remains uncertain.

Goal of the study: the aim of this prospective observational study is to investigate the prevalence of DD in a general ICU, and to value the relationship between DD and:

- expected mortality (according to SAPS-2 score)

- diagnosis on admission (multifactorial shock, cardiac arrest, respiratory failure, neurosurgical, polytrauma and septic shock)

- age

- hypertension

- gender

Materials and Methods: all patients admitted to our ICU, from January to September 2016, were part of the study, (except patients whose recovery lasted less than 24h, patients with atrial fibrillation and patients with thoracic trauma). We collected anamnestic data, vital signs, SAPS-2 score and admission diagnosis. 4 TTE views (PSLAX, PSSAX, A4C, SC) were studied; a score was assigned to each view ( 2 =optimal, $1=$ suboptimal, $0=$ unviewable) to identify the best one.

Presence/absence of DD was studied through E/A transmitralic flow (presence if $<1$ ) [Image1]; relationship between DD and hypertension, age, gender, expected mortality and admission diagnosis was analyzed. The differences between the two groups (presence/absence of DD) were analyzed with t-test, with $\chi 2$-test and fisher exact for categorical variables. Data were collected in an Excel ${ }^{\circledR}$ table and processed with a statistic package: STATA/IC 12.0.
Results: The $66,3 \%$ of our sample (59 patients) had DD.

DD resulted statistically related [Image2] with:

- Hypertension ( $\mathrm{P}=\mathbf{0 . 0 0 1 )}$

- Old age $(P<0.0001)$

- Diagnosis on admission ( $P=0.016)$

Expected mortality $(P=0.087)$ and gender $(P=0.49)$ resulted not statistically related to $\mathrm{DD}$.

The study also revealed that the best TTE views was A4C followed by PSLAX. 52,27\% of our patients had at least 2 optimal views, only $5,45 \%$ had none.

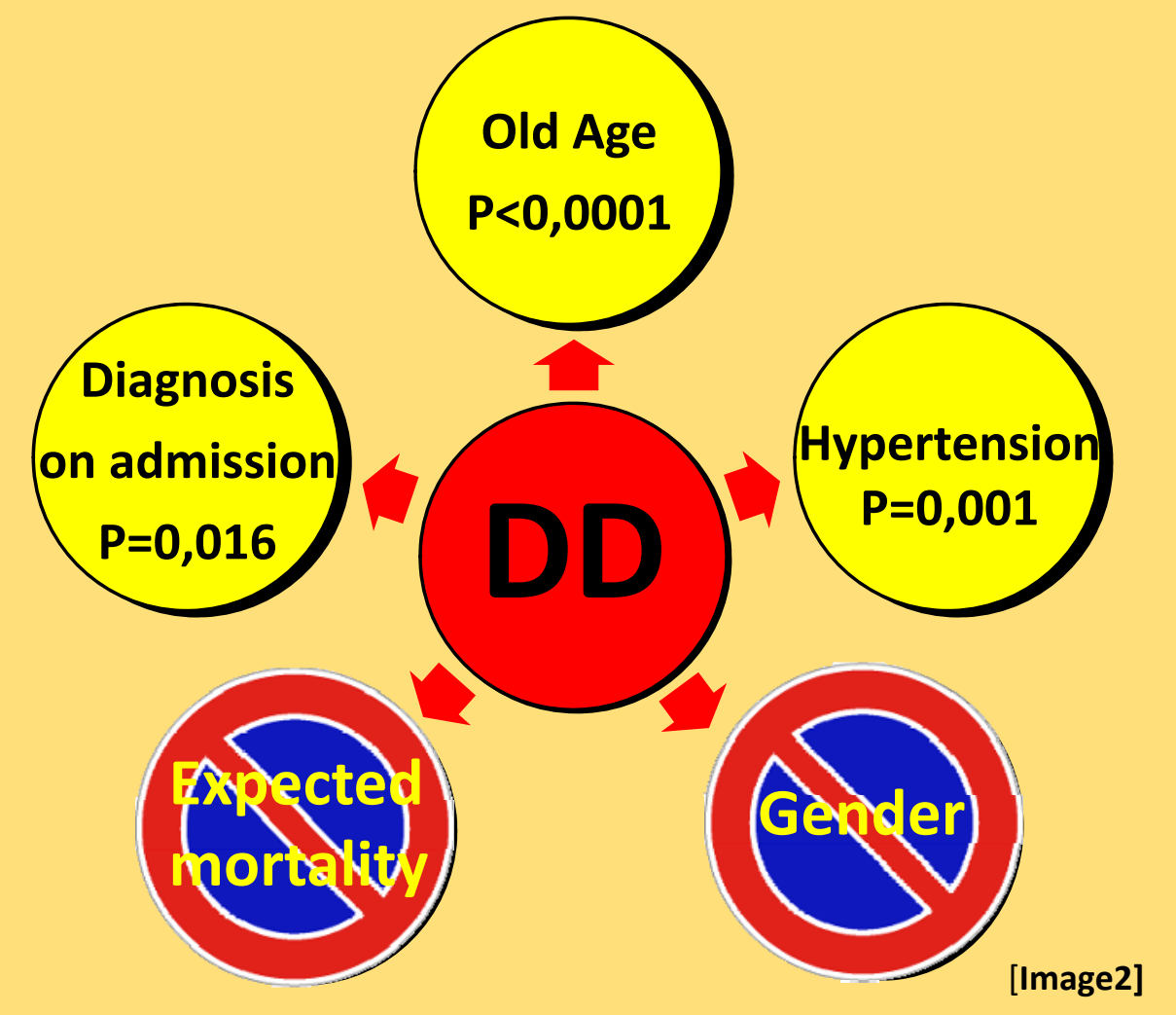

Conclusion: this study underlines that DD is a frequent cause of heart failure in ICU patients, related to old age, hypertension and mentioned diagnosis on admission.

The 2 main TTE views (PSLAX, A4C) are viewable also in mechanically ventilated and non-mobilized patients.

A bigger sample should be needed to study more detailed relations between $\mathrm{DD}$, treatments and outcome. 